\title{
Heavy ion mutagenesis combined with triclosan screening provides a new strategy for improving the arachidonic acid yield in Mortierella alpina
}

\author{
Huidan Zhang ${ }^{1,4,5}$, Dong Lu' ${ }^{2}$, Xin Li ${ }^{2}$, Yingang Feng ${ }^{1,4}$, Qiu Cui ${ }^{1,3,4}$ and Xiaojin Song ${ }^{1,4^{*}}$ (D)
}

\begin{abstract}
Background: Arachidonic acid (ARA), which is a $\omega-6$ polyunsaturated fatty acid, has a wide range of biological activities and is an essential component of cellular membranes in some human tissues. Mortierella alpina is the best strain for industrial production of ARA. To increase its yield of arachidonic acid, heavy ion beam irradiation mutagenesis of Mortierella alpina was carried out in combination with triclosan and octyl gallate treatment.
\end{abstract}

Results: The obtained mutant strain F-23 ultimately achieved an ARA yield of $5.26 \mathrm{~g} \mathrm{~L}^{-1}$, which is 3.24 times higher than that of the wild-type strain. In addition, quantitative real-time PCR confirmed that the expression levels of fatty acid synthase (FAS), $\Delta 5$-desaturase, $\Delta 6$-desaturase, and $\Delta 9$-desaturase were all significantly up-regulated in the mutant F-23 strain, especially $\Delta 6$ - and $\Delta 9$-desaturase, which were up-regulated 3- and 2-fold, respectively.

Conclusions: This study confirmed a feasible mutagenesis breeding strategy for improving ARA production and provided a mutant of Mortierella alpina with high ARA yield.

Keywords: Arachidonic acid, Desaturase, Heavy ion mutagenesis, Mortierella alpina, Octyl gallate, Triclosan

\section{Background}

Arachidonic acid (ARA), which is a $\omega-6$ polyunsaturated fatty acid, has a wide range of biological activities as it is a prerequisite for the synthesis of prostaglandins, thromboxane and leukotrienes [1]. For a long time, ARA was mainly obtained from animal tissues, such as animal livers, fish oil, and pig adrenal glands, which have low ARA contents and are limited sources [2]. Therefore, major efforts have been made to identify alternative sources of ARA. Mortierella alpina, which can accumulate a large amount of ARA in a short growth cycle, has received wide attention [3] and is considered the most prominent ARA-rich oil producer $[4,5]$. Nevertheless,

\footnotetext{
* Correspondence: songxj@qibebt.ac.cn

${ }^{1}$ Shandong Provincial Key Laboratory of Energy Genetics, Qingdao Institute of Bioenergy and Bioprocess Technology, Chinese Academy of Sciences, Qingdao 266101, Shandong, China

${ }^{4}$ Qingdao Engineering Laboratory of Single Cell Oil, Qingdao 266101,

Shandong, China

Full list of author information is available at the end of the article
}

low yield and high cost are still the bottleneck for largescale production of ARA by M.alpina.

At present, the genetic engineering method has been widely used in the increase of ARA production of $M$. alpina [6-8]. However, the metabolic regulation network of $M$. alpina is very complex $[9,10]$, changes in one or two genes are not easy to produce a transformant with the desired trait. Therefore, the mutagenesis method is still one of the effective methods to obtain high yield strains. In general, mutagenesis methods can be divided into two categories, physical mutagenesis and chemical mutagenesis. Heavy ion mutagenesis is a new physical mutagenesis technique with high bioavailability, high energy density, poor repair effects and good spatial resolution of energy deposition compared with traditional radiation sources (e.g., UV, $\gamma$ - and $\chi$-rays); thus, it can produce more extensive mutation [11-13]. As a chemical mutagenesis method, 5-fluorouracil (5-FU) is a structural analog of uracil that inhibits the synthesis of DNA and some RNA [14, 15], and it has been successfully used for 
microbial mutagenesis and cancer treatment as an effective chemical mutagen [16, 17].

Quickly identifying and screening out high-yield mutants are also important issues for breeding research. Directional screening is more effective than random selection and can reduce the amount of work [13]. From the anabolism pathway of ARA (Fig. 1), two key regulation points have been identified. The first is the synthesis of palmitic acid by fatty acid synthase (FAS), and the other is a series of desaturation reactions with stearic acid as the substrate that are catalyzed by $\Delta 9$ and $\Delta 6$ desaturase $[18,19]$. Triclosan has a broad-spectrum antibacterial effect that can inhibit the activity of FAS by inhibiting the fabI locus [20]. Mutants that grow normally on a certain concentration of triclosan plates can be identified as having relatively high FAS activity, making them good candidates for lipid production. In addition, octyl gallate is a type of antioxidant that strongly inhibits the activities of fatty acid desaturases in fungi and viruses [21-23]. The octyl gallate mechanism of action may also involve membrane interactions that lead to the loss of membrane potential and cell gap leakage. Ken-ichi Fujita observed this phenomenon when studying the inhibitory effects of octyl gallate on Zygosaccharomyces bailii and Saccharomyces cerevisiae $[24,25]$. Therefore, octyl gallate can be used as an effective reagent for screening high-yield ARA strains of M. alpina.

In this study, the wild-type strain SD003 encountered two rounds of mutagenesis and targeted screening. First, heavy ion mutagenesis was combined with triclosan to screen strains with high FAS activity. Second, strains with high desaturase activity were screened by using octyl gallate after mutagenized with 5-fluorouracil. The two-step targeted screening strategy greatly improved the screening efficiency compared with the previous method of random selection. We also investigated the mechanism by which the ARA yield is increased in the mutagenized strain as well as the batch fermentation conditions for optimal production.

\section{Methods}

\section{Strains and culture conditions}

In the present study, M. alpina SD003 (CGMCC No. 7960 ) was used as the initial strain. GY medium (glucose, $20 \mathrm{~g} \mathrm{~L}^{-1}$; yeast extract, $10 \mathrm{~g} \mathrm{~L}^{-1}$; and agar, $20 \mathrm{~g} \mathrm{~L}^{-1}$ ) was used to screen $M$. alpina. The seed culture medium contained glucose, $30 \mathrm{~g} \mathrm{~L}^{-1}$; yeast extract, $6 \mathrm{~g} \mathrm{~L}^{-1}$; $\mathrm{KH}_{2} \mathrm{PO}_{4}, 3 \mathrm{~g} \mathrm{~L}^{-1} ; \mathrm{NaNO}_{3}, 2.8 \mathrm{~g} \mathrm{~L}^{-1}$; and $\mathrm{MgSO}_{4} \cdot 7 \mathrm{H}_{2} \mathrm{O}$, $0.5 \mathrm{~g} \mathrm{~L}^{-1}$. The medium for the shake-flask and bioreactor fermentation cultures contained glucose, $80 \mathrm{~g} \mathrm{~L}^{-1}$; yeast extract, $11 \mathrm{~g} \mathrm{~L}^{-1} ; \mathrm{KH}_{2} \mathrm{PO}_{4}, 3.8 \mathrm{~g} \mathrm{~L}^{-1}$; $\mathrm{NaNO}_{3}, 3.5 \mathrm{~g} \mathrm{~L}^{-1}$; and $\mathrm{MgSO}_{4} \cdot 7 \mathrm{H}_{2} \mathrm{O}, 0.5 \mathrm{~g} \mathrm{~L}^{-1}$. The seed cultures were grown at $25{ }^{\circ} \mathrm{C}$ with shaking at $200 \mathrm{rpm}$ for $2 \mathrm{~d}$ and were then inoculated into shake flasks at $10 \%(\mathrm{v} / \mathrm{v})$ inoculum and cultured at $25{ }^{\circ} \mathrm{C}$ with shaking at $200 \mathrm{rpm}$ for $7 \mathrm{~d}$. The bioreactor cultivations were performed in 5-L Biostat B plus bioreactors (Sartorius Stedim Biotech, Germany) containing $3 \mathrm{~L}$ of medium at $25{ }^{\circ} \mathrm{C}$ with stirring at $200 \mathrm{rpm}$ for $10 \mathrm{~d}$. Triclosan, octyl gallate, and 5fluorouracil were purchased from Sangon Biotech Shanghai Co., Ltd. (Shanghai, China), Shanghai Yuanye Biological Technology Co., Ltd. and Alfa Aesar (China) Chemical Co., Ltd., respectively.

\section{Heavy ion beam irradiation and triclosan screening}

To obtain a stable, high-yield ARA strain, the overall experiment included a four-step screening program with gradually increased screening efficiency, as shown in Fig. 2. The detailed process is described below.

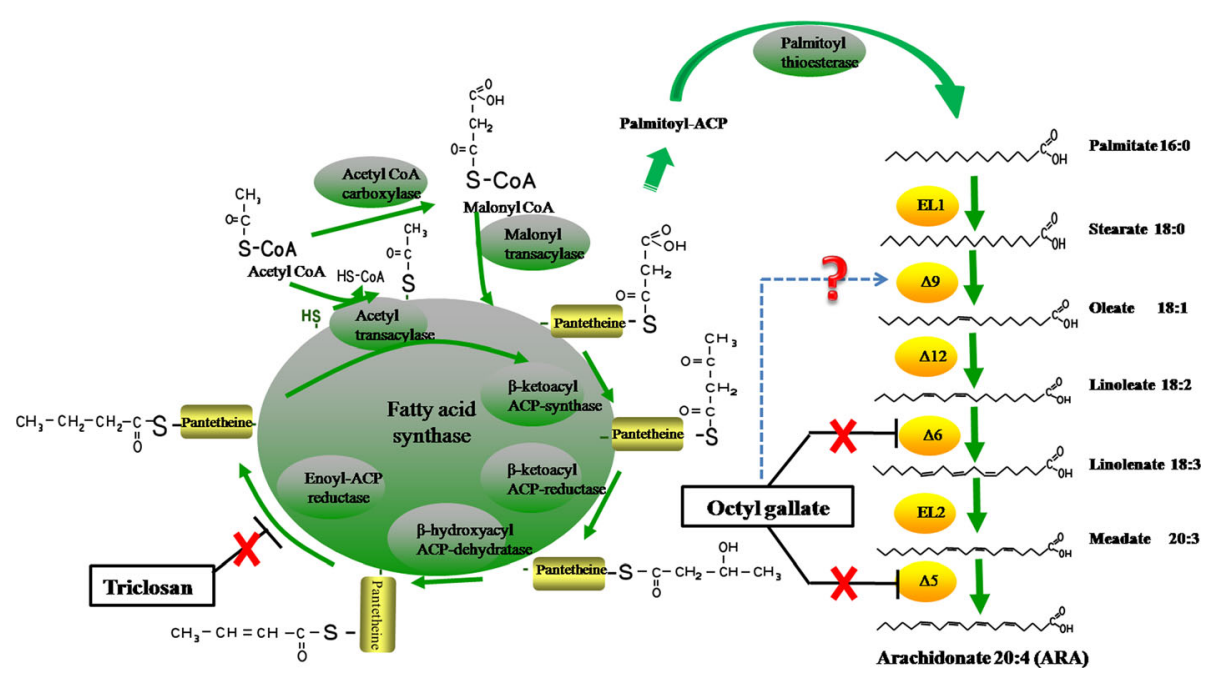

Fig. 1 The ARA biosynthesis pathway and the action sites of inhibitors in Mortierella alpina 


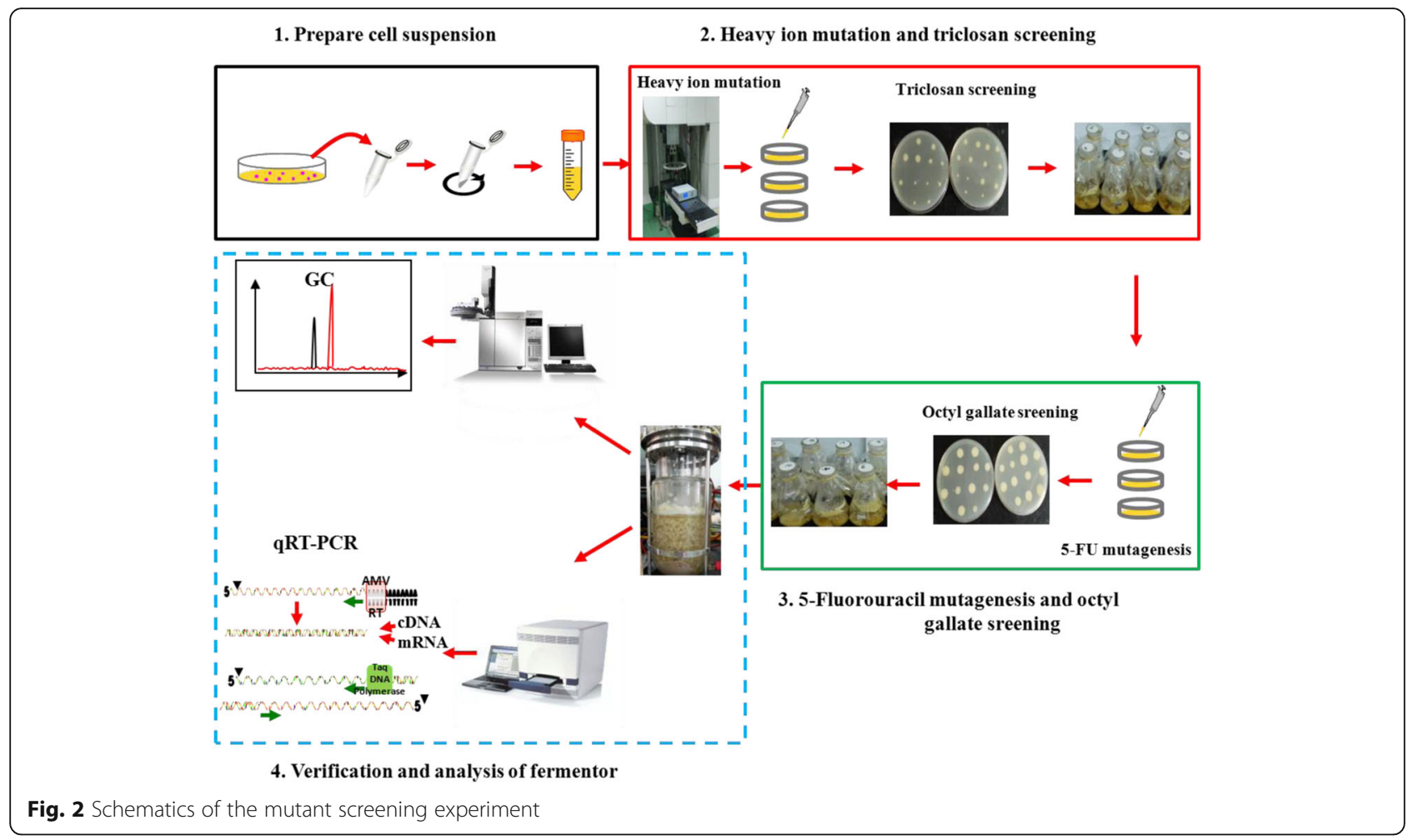

The first step was the preparation of the cell suspension. Spores of M. alpina SD003 were rinsed with sterile water and filtered through three layers of sterile lens paper. The spore suspension was centrifuged at $1900 \times \mathrm{g}$ for $10 \mathrm{~min}$ and diluted with sterile water to a concentration of $10^{6}$ spores $\mathrm{mL}^{-1}$.

Then, the heavy ion beam irradiation experiments were carried out at the Heavy Ion Research Facility in Lanzhou (HIRFL) at the Institute of Modern Physics at the Chinese Academy of Sciences. $\mathrm{A}^{12} \mathrm{C}^{6+}$ heavy ion beam was used with an energy of $80 \mathrm{MeV}^{-1}$ and an LET (the energy transferred per unit length) of $31 \mathrm{keV} \mu \mathrm{m}^{-1}$. The heavy ion irradiation dose was $0,40,80,120,180$, or 240 Gy. The irradiated spore suspension was diluted to $10^{3}$ and $10^{4}$ spores $\mathrm{mL}^{-1}$, and $100 \mu \mathrm{L}$ of the spore suspensions were coated on GY plate culture medium. Each dilution was performed in triplicate. After incubation in the dark at $25{ }^{\circ} \mathrm{C}$ for $3 \mathrm{~d}$, the mortality rate was calculated using the unirradiated spore suspension as a control.

To determine the optimal antimicrobial concentration of triclosan, the unirradiated spore suspension was treated with different triclosan concentrations $(0,0.1,0.5$, $0.6,0.8,1.0,1.5,2,2.5$ and $3 \mathrm{mgL}^{-1}$ ) on GY plates at $25^{\circ} \mathrm{C}$ for $3 \mathrm{~d}$, and the mortality rate was calculated. After determining the optimum concentration, the irradiated spores were properly diluted and incubated on plates containing this concentration of triclosan. After the passage of three generations, stable mutants were cultured and tested for ARA production. Consequently, the strain with the highest ARA yield, mutant Z-44, was selected for the next round of 5-fluorouracil mutagenesis.

\section{5-fluorouracil mutagenesis and octyl gallate screening} 5-fluorouracil mutagenesis was carried out as the third step of the breeding experiment. The mutant Z-44 obtained from the preceding steps was cultured in PDA medium for $10 \mathrm{~d}$ and then washed with $0.9 \% \mathrm{NaCl}$ to prepare a spore suspension. One milliliter of the spore suspension $\left(10^{8}\right.$ cells) was inoculated into a $50-\mathrm{mL}$ Erlenmeyer flask containing $10 \mathrm{~mL}$ of $0.9 \% \mathrm{NaCl}$. After incubation for $12 \mathrm{~h}$ at $25{ }^{\circ} \mathrm{C}$ with shaking at $120 \mathrm{rpm}$, 5-fluorouracil was added to this starvation medium at a final concentration of $20 \mu \mathrm{g} \mathrm{mL}{ }^{-1}$. The mixture was incubated in a rotary shaker for $5 \mathrm{~h}, 10 \mathrm{~h}$ and $36 \mathrm{~h}$ at $25^{\circ} \mathrm{C}$ with shaking at $120 \mathrm{rpm}$.

After 5-fluorouracil mutagenesis, the mutated spores were spread onto GY plates containing $14 \mu \mathrm{g} \mathrm{mL} L^{-1}$ of octyl gallate.

\section{Determination of the genetic stability of the mutant}

Mutant strains were passaged for 10 generations on PDA slants. Changes in the ARA production performance indexes of the strains were analyzed before and after passage. 


\section{Analytical methods}

\section{Determination of cell dry weight}

Fermentation broth was filtered at room temperature, and the mycelia were washed twice with distilled water. The cell pellets were dried to a constant weight at $50{ }^{\circ} \mathrm{C}$.

\section{Total lipid extraction and fatty acid determination}

Lipid extraction and transesterification were carried out according to a previously described method [26]. Total lipids were extracted using approximately $100 \mathrm{mg}$ of mycelia (dry weight). Fatty acid methyl esters (FAMEs) were obtained by reacting the lipids with $2 \%$ sulfuric acid in methanol $(v / v)$ at $85{ }^{\circ} \mathrm{C}$ for $2.5 \mathrm{~h}$. FAMEs were extracted with $\mathrm{n}$-hexane and analyzed using a gas chromatograph (Agilent Technologies) equipped with an HP-INNOWAX (30 $\mathrm{m} \times 0.25 \mathrm{~mm}, 0.25 \mu \mathrm{m}$ film thickness) capillary column. The oven temperature was set to $100{ }^{\circ} \mathrm{C}$ for $1 \mathrm{~min}$ and was then raised to $250{ }^{\circ} \mathrm{C}$ at a rate of $15{ }^{\circ} \mathrm{C}$ per min before being allowed to stand at $250{ }^{\circ} \mathrm{C}$ for $5 \mathrm{~min}$. Peak detection was performed using a flame ionization detector (FID). The carrier gas was nitrogen, and the split ratio was 1:19. The injection volume was $1 \mu \mathrm{L}$.

\section{Quantitative real-time PCR ( $q R T-P C R$ ) analysis}

After $7 \mathrm{~d}$ of fermentation, total RNA was extracted with an RNeasy Plant Mini Kit (QIAGEN, Germany) according to the manufacturer's protocol. cDNA was prepared using a Revert Aid First Strand cDNA Synthesis Kit (Thermo Scientific), and the FastStart Universal SYBR Green Master Mix (ROX) was used for the PCR reactions. The qRT-PCR primers are listed in Table 1. Differences in gene expression were detected using a LightCycler R480 Real-time Detection System (Roche). 18SRNA was used as an internal control to normalize the expression levels. The comparison of CT method $\left(2^{-\Delta \Delta C T}\right)$ was used to analyze the gene expression differences.

Table 1 Primers used in this study

\begin{tabular}{|c|c|c|}
\hline Primers & Sequence $\left(5^{\prime}-3^{\prime}\right)$ & Description \\
\hline 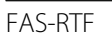 & TCCTITCTATGGTGCTTCCTCA & \\
\hline FAS-RTR & ATCTTACCTTGCTGCCGTTCT & FAS RT-qPCR \\
\hline$\Delta 5-R T F$ & TGTCTGGAAGATTCTGGGAGC & \\
\hline$\Delta 5-R T R$ & TTGGTTGGGCTTGATACGAC & $\triangle 5 \mathrm{RT}-\mathrm{qPCR}$ \\
\hline$\Delta 6-R T F$ & TGTTCTGGCAGCAGTGCGGATGG & \\
\hline$\Delta 6-R T R$ & GGCGTGGTGAGTGTTGTGCTTGTCC & $\triangle 6 \mathrm{RT}-\mathrm{qPCR}$ \\
\hline$\Delta 9-R T F$ & TCGTTCAGTGGCAGCACAAGA & \\
\hline$\Delta 9-R T R$ & CAAGACGGAGGATAGCAGCATA & $\triangle 9$ RT-qPCR \\
\hline$\Delta 12-R T F$ & TGGGTGCTGGCTCACGAGTGT & \\
\hline$\Delta 12-R T R$ & CCAGTGGCCTTGTGGTGCTTC & $\triangle 12$ RT-qPCR \\
\hline 18S-RTF & CGTACTACCGATTGAATGGCTTAG & \\
\hline 18S-RTR & CCTACGGAAACCTTGTTACGACT & $\begin{array}{l}\text { Internal control for } \\
\text { RT-qPCR }\end{array}$ \\
\hline
\end{tabular}

\section{Results}

Heavy ion beam irradiation-induced $M$. alpina mortality Heavy ion mutagenesis has been successfully applied to plants $[27,28]$ and some industrial microbial mutagenesis breeding programs $[11,29]$, but as far as we know, this study is the first to use it with $M$. alpina. Due to a lack of reference conditions for the heavy ion mutagenesis of $M$. alpina, we investigated the lethality of five doses of heavy ion beam irradiation in this experiment. As shown in Fig. 3, at 40, 80, 120, 180 and 240 Gy, the lethality rates were $13 \%, 17 \%, 25 \%, 37 \%$, and $71 \%$, respectively. The lethality of spores increased as the dose increased, with a sharp increase to $71 \%$ at $240 \mathrm{~Gy}$. In general, a lethality rate of $70-80 \%$ yields a high positive mutation rate [30]. Therefore, the heavy ion beam irradiation mutagenesis was performed at a 240 Gy dose in this study.

\section{Screening of high-yield ARA mutants with triclosan}

Determining how to screen high-yield strains efficiently from a large number of mutants is the key issue in mutagenesis breeding experiments. When traditional shake flask fermentations are used as the means of screening, the screening cycle is long and troublesome. However, when triclosan is used as the screening method, the screening scope is much narrower, and the screening efficiency is greatly improved.

To determine the susceptibility of the wild-type strain to triclosan, M. alpina SD003 was spread onto GY plates containing different concentrations of triclosan. Triclosan was found to strongly inhibit the growth of the wildtype strain. The number of colonies decreased with increasing triclosan concentrations. The lethal rate was approximately $92.8 \%$ when the triclosan concentration was $2 \mathrm{mg} \mathrm{L}^{-1}$. Thus, $2 \mathrm{mg} \mathrm{L}^{-1}$ of triclosan was selected as a suitable concentration for screening mutants after heavy ion beam irradiation mutagenesis.

The spore suspensions treated with a heavy ion beam irradiation dose of 240 Gy were properly diluted, spread onto GY plates containing $2 \mathrm{mg} \mathrm{L}^{-1}$ triclosan and incubated at $25{ }^{\circ} \mathrm{C}$ for $3 \mathrm{~d}$. Only mutant strains with high FAS activity could grow and form large colonies. We randomly selected 120 large colonies and screened them three times on GY plates containing $2 \mathrm{mg} \mathrm{L}^{-1}$ triclosan. Fifty-three mutants were still able to grow steadily. As shown in Fig. 4, 20 strains with high lipid and ARA contents were further screened via shake flask fermentations. The mutant Z-44 had the highest ARA production, with lipid and ARA yields of $9.65 \mathrm{~g} \mathrm{~L}^{-1}$ and $3.80 \mathrm{~g} \mathrm{~L}^{-1}$, which were 1.10 times and 2.06 times higher than that of the wild-type strain, respectively (Table 2). The mutant strain Z-44 mainly had an increased total lipid content (38.3\% of the biomass), which was nearly double that of the wild-type strain (19.8\%). Analyzing the fatty acid composition (Table 3) revealed that the 


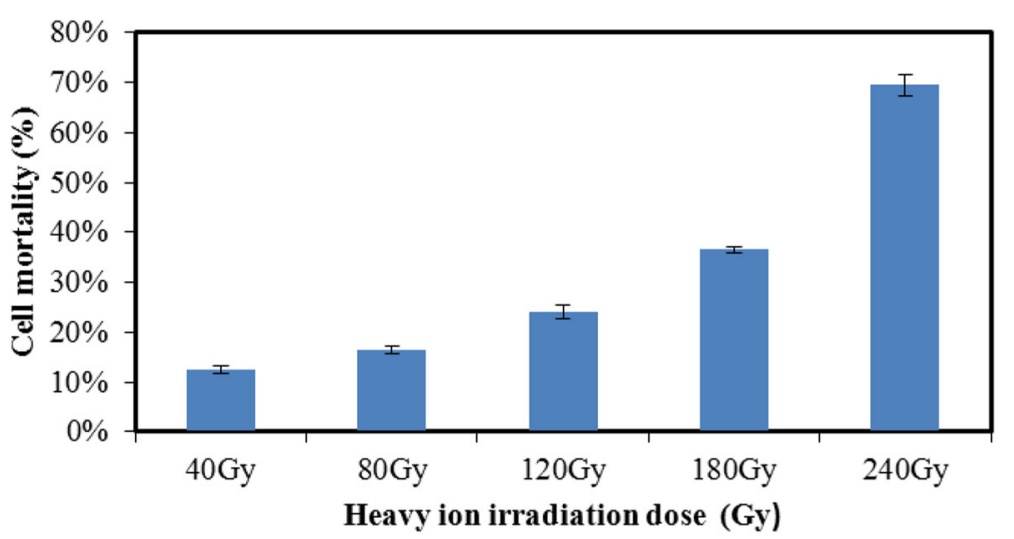

Fig. 3 Mortality of Mortierella alpina SD003 treated with different doses of heavy ion beam irradiation. Data are the means of three replicate experiments, and the error bars indicate standard deviation

ARA content of mutant Z-44 was significantly improved (from $27.04 \%$ to $39.37 \%$ ) and that the ratio of $\mathrm{C} 16: 0$ and C18:1 decreased significantly.

\section{5-fluorouracil mutagenesis and octyl gallate screening of strains}

To further improve the yield of ARA, the mutant strain Z-44 was used as the starting strain for the second round of mutagenesis with 5-fluorouracil, which is a compound similar to uracil that can be converted into effective fluorouracil deoxynucleotides that interfere with the synthesis of DNA. First, we studied the lethality of 5fluorouracil after different treatment times. The results showed that the lethality rate was $50 \%$ at $5 \mathrm{~h}, 71 \%$ at $10 \mathrm{~h}$ and $93 \%$ at $36 \mathrm{~h}$ for $20 \mu \mathrm{g} \mathrm{mL} \mathrm{m}^{-1} 5$-fluorouracil treatment. Therefore, considering the operability of the experiment, we ultimately selected $10 \mathrm{~h}$ as the processing time.
Alkyl gallate salts have been reported to be antioxidants with strong inhibitory effects on the $\Delta 6$-fatty acid desaturase in the ARA-producing fungi M. alpina 1S-4, and the inhibition of octyl gallate was the most obvious [25]. Therefore, strains resistant to octyl gallate may have high fatty acid desaturase activities, thereby promoting the formation of ARA. The lethal rate of octyl gallate at

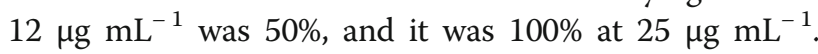
The lethal rate approached $86 \%$ when the spores were spread on GY plates containing $12 \mu \mathrm{g} \mathrm{mL}^{-1}$ octyl gallate after treatment with 5 -fluorouracil for $10 \mathrm{~h}$. After 3 rounds of screening, 80 strains were selected for shake flask fermentation validation.

The ARA yield of the mutant strain F-23 was $5.26 \mathrm{~g} \mathrm{~L}^{-1}$, which was the highest yield observed in the mutants and 3.24 times higher than that of the wild-type strain $\left(1.24 \mathrm{~g} \mathrm{~L}^{-1}\right)$. The ARA content of the mutant F-23 was $49.08 \%$, which was $81.51 \%$ higher than that of the

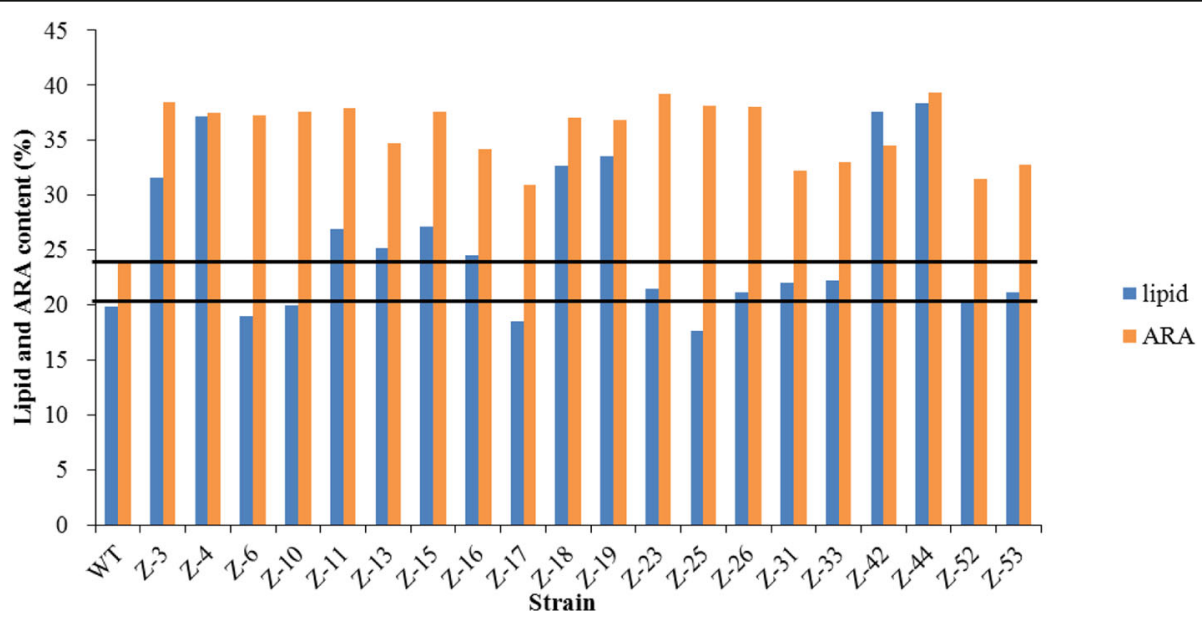

Fig. 4 The total lipid and ARA contents of the isolated mutants in $250 \mathrm{~mL}$ shake flask after heavy ion beam irradiation mutagenesis. The initial glucose concentration was $80 \mathrm{~g} \mathrm{~L}^{-1}$. "WT" indicates the wild-type strain. Numbers from Z-3 to Z-53 indicate the mutants 
Table 2 Productivity of wild-type strain, mutant strain Z-44 and F-23

\begin{tabular}{llllll}
\hline Strains & $\begin{array}{l}\text { Biomass } \\
\left(\mathrm{g} \mathrm{L}^{-1}\right)\end{array}$ & $\begin{array}{l}\text { Lipid content } \\
(\%)\end{array}$ & $\begin{array}{l}\text { Lipid yield } \\
\left(\mathrm{g} \mathrm{L}^{-1}\right)\end{array}$ & $\begin{array}{l}\text { ARA content } \\
(\%)\end{array}$ & $\begin{array}{l}\text { ARA yield } \\
\left(\mathrm{g} \mathrm{L}^{-1}\right)\end{array}$ \\
\hline WT & $23.24 \pm 0.46$ & $19.8 \pm 0.36$ & $4.60 \pm 0.56$ & $27.04 \pm 0.63$ & $1.24 \pm 0.18$ \\
Z-44 & $25.2 \pm 0.93$ & $38.3 \pm 0.97$ & $9.65 \pm 0.26$ & $39.37 \pm 0.28$ & $3.80 \pm 0.65$ \\
F-23 & $28.2 \pm 0.36$ & $38.0 \pm 0.25$ & $10.72 \pm 0.41$ & $49.08 \pm 0.17$ & $5.26 \pm 0.48$ \\
\hline
\end{tabular}

wild-type strain (27.04\%) and $24.66 \%$ higher than that of the mutant Z-44. Analysis of the fatty acid composition revealed that the $\mathrm{C} 18: 0$ ratio was decreased significantly due to the rising catalytic capacity of the $\Delta 9$-desaturase (Fig. 5). The growth characteristics of the wild-type and two mutant strains, Z-44 and F-23, are shown in Tables 2 and 3.

Expression levels of the endogenous FAS and $\Delta 5, \Delta 6, \Delta 9$ and $\Delta 12$-desaturase genes in the wild-type and mutant strains

To confirm the roles of triclosan and octyl gallate in the fatty acid synthesis pathway of $M$. alpina, five genes, fatty acid synthase, $\Delta 5$-desaturase, $\Delta 6$-desaturase, $\Delta 9$ desaturase, and $\Delta 12$-desaturase, that are related to ARA synthesis were analyzed by qRT-PCR at the mRNA level. As shown in Fig. 5, the mRNA expression level of FAS in mutant Z-44 was significantly up-regulated at a level 4.4 times higher than that of the wild-type strain. Therefore, the fatty acid synthase was the main locus of action of triclosan; some other studies have also demonstrated that triclosan inhibited the activity of enoyl-ACP reductase, which is a module of the FAS complex [19, 31, 32].

For the mutant F-23, which is a further mutagenized strain of the mutant Z-44, not only the expression level of FAS was increased by 3.1 times but the expression levels of all of the desaturases were also up-

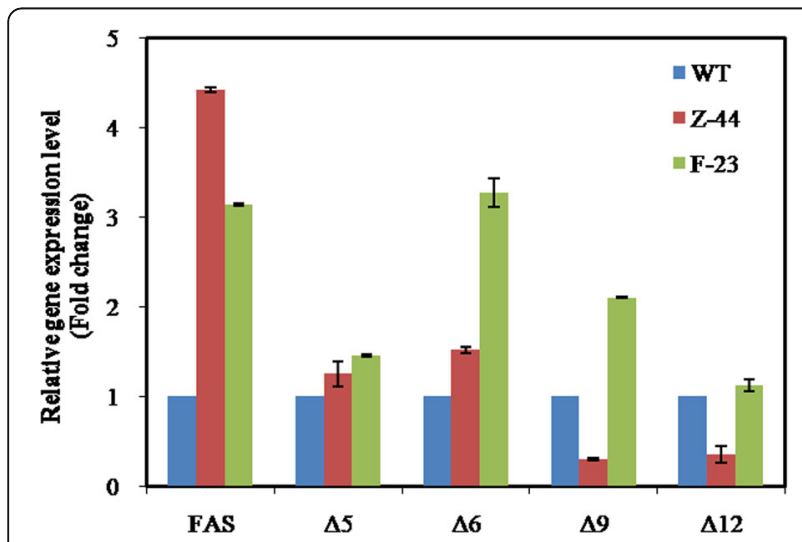

Fig. 5 Relative gene expression levels of fatty acid synthase (FAS), $\Delta 5$ - desaturase, $\Delta 6$-desaturase, $\Delta 9$ - desaturase and $\Delta 12$ - desaturase of the wild-type, mutant Z-44 and mutant F-23 strains regulated, especially the $\Delta 6$ and $\Delta 9$-desaturases, which were significantly up-regulated by 3.2 and 2.1 times, respectively, compared to those of the wild-type strain (Fig. 5).

\section{Genetic stability of the mutant}

To investigate the genetic stability of the mutagenized strain, strain F-23 was cultured for 10 generations. Fermentation was carried out every five generations, and the biomass, lipid and ARA yields were measured three times. As shown in Table 4, the ARA yield was approximately $5.5 \mathrm{~g} \mathrm{~L}^{-1}$, and the biomass was stable at approximately $29 \mathrm{~g} \mathrm{~L}^{-1}$ after 10 generations, indicating that the mutant F-23 had good genetic stability.

\section{Batch fermentation of mutant F-23}

The mutant F-23 was further evaluated in batch fermentations in a 5-L bioreactor. The growth curves of F-23 and wild-type SD003 are shown in Fig. 6. The growth of the mycelia can be seen to experience three typical periods: delay, rapid growth and decay. The mutant F-23 had a relatively longer delay period (approximately $4 \mathrm{~d}$ ) than wild-type SD003, possibly due to effects of mutagenesis on the expression of some genes or enzymes in vivo, resulting in a growth delay. The glucose consumption rate of both strains was basically the same. Subsequently, a more efficient logarithmic phase was observed in mutant F-23, which had a final biomass of $32.5 \mathrm{~g} \mathrm{~L}^{-1}, 14.04 \%$ higher than strain SD003. Finally, the decay period was observed in both of these strains after $8 \mathrm{~d}$, and it was more obvious in the wild-type strain.

The total lipid and ARA yields almost synchronously accumulated with mycelial growth. After $10 \mathrm{~d}$ of fermentation, the lipid yield of mutant F-23 reached a maximum of $10.93 \mathrm{~g} \mathrm{~L}^{-1}, 1.55$ times of that of wild-type strain SD003 (7.06 $\left.\mathrm{g} \mathrm{L}^{-1}\right)$. Meanwhile, the ARA yield of mutant F-23 was $5.46 \mathrm{~g} \mathrm{~L}^{-1}, 2.22$ times of that of strain SD003 $\left(2.45 \mathrm{~g} \mathrm{~L}^{-1}\right)$. These results indicate that mutant F-23 could be a valuable candidate strain for producing ARA.

\section{Discussion}

Due to the lack of markers and low transformation frequencies, there are still some technical problems in 
Table 3 Analysis of fatty acid composition of wild-type strain and mutant strains

\begin{tabular}{|c|c|c|c|c|c|c|c|c|}
\hline \multirow[t]{2}{*}{ Strains } & \multicolumn{8}{|c|}{ Fatty acids content (\% total fatty acids) } \\
\hline & $\mathrm{C} 14: 0$ & C16:0 & C18:0 & C18:1 & $\mathrm{C} 18: 2$ & C18:3 & C20:3 & C20:4 \\
\hline WT & $2.83 \pm 0.07$ & $18.33 \pm 0.46$ & $13.32 \pm 0.99$ & $25.65 \pm 1.79$ & $5.72 \pm 0.52$ & $2.88 \pm 0.14$ & $3.75 \pm 0.12$ & $23.99 \pm 1.92$ \\
\hline Z-44 & $1.13 \pm 0.04$ & $11.26 \pm 0.93$ & $22.35 \pm 1.37$ & $7.91 \pm 0.27$ & $6.45 \pm 0.16$ & $3.83 \pm 0.05$ & $2.64 \pm 0.09$ & $39.37 \pm 0.28$ \\
\hline $\mathrm{F}-23$ & $0.61 \pm 0.01$ & $10.93 \pm 0.02$ & $13.95 \pm 0.01$ & $8.48 \pm 0.29$ & $5.35 \pm 0.12$ & $3.97 \pm 0.07$ & $3.53 \pm 0.02$ & $49.12 \pm 0.06$ \\
\hline
\end{tabular}

Data are expressed as means \pm standard deviations

the simultaneous transformation of several genes of M. alpina by molecular methods. The mutagenesis breeding method is still an effective breeding method, because it could change many characteristics without requiring much biochemical and genetic information of the strain. In this study, heavy ion beam irradiation mutagenesis combined with directional screening was carried out and greatly improved the work efficiency. This is the first report about applying heavy-ion irradiation mutagenesis on M. alpina breeding.

So far, researchers have carried out a variety of ways to mutagenize on $M$. alpina, such as UV, $\gamma$ - and $\mathrm{X}$-rays, etc. [33]. Compared with the traditional physical mutagenesis (e.g., UV, $\gamma$ - and $\chi$-rays), heavy ion beam irradiation mutagenesis has higher LET (the energy transferred per unit length) that can induce more biological damage than other types of radiation, which is difficult to repair. Therefore, mutations caused by heavy ion mutagenesis are relatively stable [34]. The LET value of the heavy beam used in this study is $31 \mathrm{keV} \mu \mathrm{m}^{-1}$, while the LET values of the $x$-ray and $\gamma$-ray are only 0.2 and 2 . $0 \mathrm{keV} \mu \mathrm{m}^{-1}$ respectively [35]. On the other hand, the heavy ion ionization peak (Bragg peak) is sharper. Thus, the interaction area between the heavy ion beam and the biological sample is more concentrated, which results in a higher mutation rate [36]. Therefore, heavy ion mutagenesis can be used for M. alpina as a more efficient breeding method.

Usually, a lot of works are needed during the mutants screening. So, in order to reduce the workload, this study attempts to establish a rapid pre-screening method. Triclosan has been used in some studies on lipid accumulation [12], but whether triclosan could improve ARA production is not clear. From our results, using the triclosan screening method, twenty strains screened from all the 53 mutants had an ARA content increasing by at least $30 \%$, with the highest ARA content of mutant Z-44 increased nearly $40 \%$. The positive mutation rate is $38 \%$, which is higher than other methods and greatly improves the work efficiency. These results indicated that the method using heavy ion mutagenesis combined with triclosan screening was an effective method for improving ARA production of M. alpina.

In order to investigate the effect of triclosan on lipid and ARA synthesis, we used qRT-PCR to investigate the mRNA expression levels of desaturase and fatty acid synthase (FAS) in M. alpina. The results showed that the mRNA expression level of FAS in mutant Z-44 was significantly increased (4.4 times), indicating that triclosan strongly affected the FAS activity, and the selected mutant had strong FAS activity (Fig. 5). This is also consistent with the fatty acid composition of Z-44 in Table 3. The contents of palmitic acid (C16:0) in mutant Z-44 were significantly decreased, and the proportion of stearic acid (C18:0) in the total fatty acids is still high. This phenomenon may cause by the increase of FAS activity and the low expression levels of the four desaturases, especially the low expression of $\Delta 9-, \Delta 12$-desaturase, which still blocked the ARA biosynthesis (Figs. 1 and 5). Therefore, the enhancement of desaturase expressions or activities were selected as the second round screening strategy.

According to the previous report, octyl gallate can strongly inhibit the activity of $\Delta 5$ - and $\Delta 6$ - desaturase of $M$. alpina [37], so we decided to use it as the screening agent. The results of qRT-PCR showed that the mRNA expression levels of all the desaturases of mutant F-23 were improved, especially the expression levels of $\Delta 9$ - and $\Delta 6$-desaturases, which suggested that the main action sites of octyl gallate may be the $\Delta 6$ and $\Delta 9$ - desaturase. These results suggested that octyl

Table 4 Genetic stability of mutant strain F-23

\begin{tabular}{llllll}
\hline Passage number & $\begin{array}{l}\text { Biomass } \\
\left(\mathrm{g} \mathrm{L}^{-1}\right)\end{array}$ & $\begin{array}{l}\text { Lipid content } \\
(\%)\end{array}$ & $\begin{array}{l}\text { Lipid yield } \\
\left(\mathrm{g} \mathrm{L}^{-1}\right)\end{array}$ & $\begin{array}{l}\text { ARA content } \\
(\%)\end{array}$ & $\begin{array}{l}\text { ARA yield } \\
\left(\mathrm{g} \mathrm{L}^{-1}\right)\end{array}$ \\
\hline 1 & $29.20 \pm 0.64$ & $38.97 \pm 0.26$ & $11.37 \pm 0.17$ & $50.36 \pm 0.57$ & $5.73 \pm 0.15$ \\
5 & $28.52 \pm 0.39$ & $37.06 \pm 1.07$ & $10.57 \pm 0.45$ & $48.39 \pm 0.72$ & $5.12 \pm 0.29$ \\
10 & $28.93 \pm 0.83$ & $38.25 \pm 0.35$ & $11.06 \pm 0.22$ & $49.12 \pm 0.06$ & $5.43 \pm 0.11$ \\
\hline
\end{tabular}




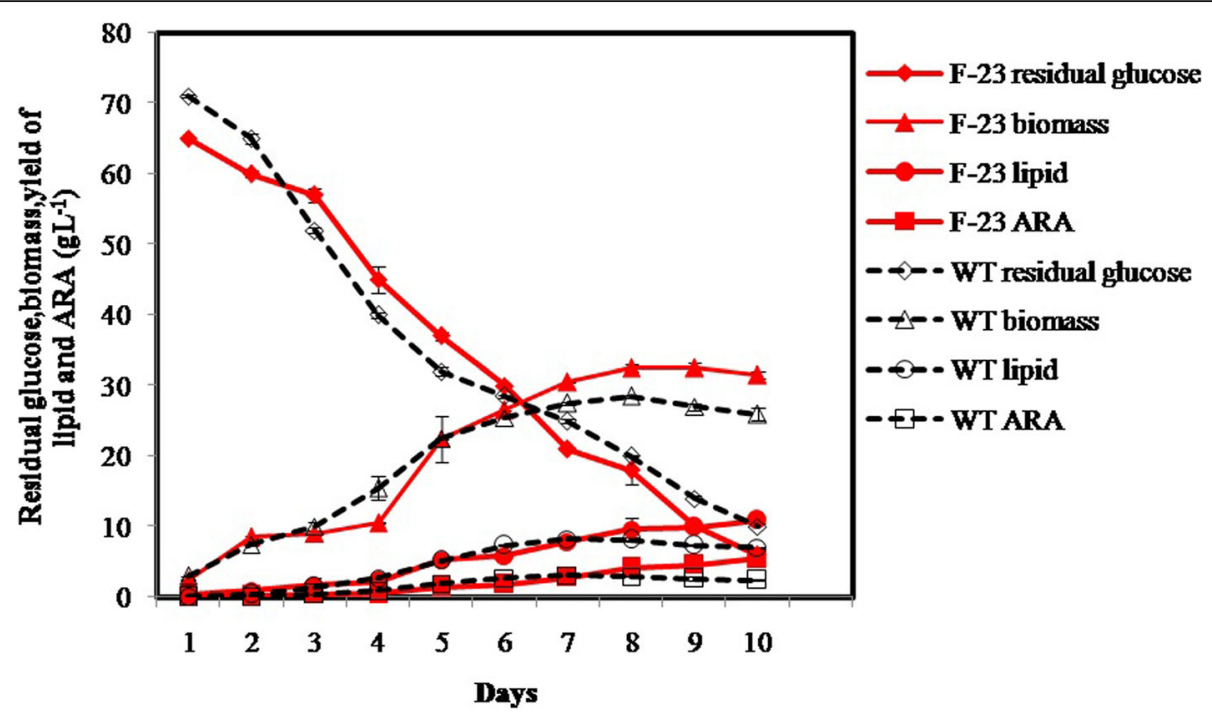

Fig. 6 Growth curves of the mutant F-23 and wild-type strains in 5-L fermenters. $80 \mathrm{~g} \mathrm{~L}^{-1}$ glucose was supplemented to the medium once a day from the first day. Red represents the mutant F-23 strain, and black represents the wild-type strain

gallate can inhibit the activity of not only $\Delta 5$ - and $\Delta 6$ desaturases, but also the other desaturases, especially $\Delta 9$-desaturase (Fig. 1). In addition, compared with the Z-44 mutant, the content of C18: 0 and C18: 2 in mutant F-23 were decreased significantly. In particular, the content of $18: 0$ decreased from $22.35 \%$ to $13.95 \%$, with a drop of $37.58 \%$. This may be due to the activity increase of the $\Delta 9-, \Delta 6$ - desaturase, which increases the flux of ARA biosynthesis, thereby further increasing the ARA content (from $38.37 \%$ to $49.12 \%$ ).

Our results showed that compared with the wild type, the lipid and ARA yield of mutant F-23 were obviously increased 1.33 and 3.24 times, respectively. The genetic trait of mutant F-23 was stable during 10 passages. Based on our results, the ARA yield will be further improved, if we next carry out the fermentation optimization and the genetic transformation methods, such as the overexpression of $\mathrm{VHb}, \mathrm{ME}$ and other genes [38-41]. In general, our study provides a good method and strategy for screening microorganisms with high yield of unsaturated fatty acids.

\section{Conclusions}

In conclusions, the improvement of the industrial strain was an effective way to reduce the ARA production costs. In this study, mutant F-23 was selected after heavy ion beam irradiation combined with triclosan and octyl gallate treatment. Compared with the wild-type strain, the total lipid and ARA yields were increased by 1.33 and 3.24 times, respectively. These results provide a good method and strategy for screening microorganisms with high yield of unsaturated fatty acids.

\section{Abbreviations}

5-FU: 5-fluorouracil; ARA: arachidonic acid; CGMCC: China General Microbiological Culture Collection Center; DGLA: dihomo- - -linolenic acid: FAMEs: fatty acid methyl esters; FAS: fatty acid synthase; FID: flame ionization detector; GLA: Y-linolenic acid; GY: screening medium; HIRFL: Heavy lon Research Facility in Lanzhou; LA: linoleic acid; LET: the energy transferred per unit length; M. alpina: Mortierella alpina; ME: malic acid; PDA: potato dextrose agar medium; VHb: Vitreoscilla hemoglobin

\section{Acknowledgements}

We are very grateful to Dr. Dong Lu, Jie Wang and Xin Li from the Institute of Modern Physics at the Chinese Academy of Sciences for their help with the heavy ion beam irradiation mutagenesis experiments.

\section{Funding}

This work is financially supported by the National Key Research and Development Program (no. 2016YFA0601400), Key research and development program of Shandong Province (no. 2017YYSP026), Qingdao independent innovation major project (16-7-1-11-zdzx-nsh). This study is a contribution to the international IMBER project. The project was supported by Shandong Provincial Key Laboratory of Energy Genetics.

\section{Availability of data and materials}

All data generated or analyzed during this study are included in this published article.

\section{Authors' contributions}

$\mathrm{HZ}$ performed the experiments as part of her doctoral work. All this work was carried out under the supervision of QC and XS. DL and XL performed the heavy ion beam irradiation experiments. XS assisted in the bioreactor experiment. QC and YF also assisted in editing the manuscript. All the authors have read and approved the final manuscript.

Ethics approval and consent to participate Not applicable.

\section{Competing interests}

The authors declare that they have no competing interests.

\section{Publisher's Note}

Springer Nature remains neutral with regard to jurisdictional claims in published maps and institutional affiliations. 


\section{Author details}

'Shandong Provincial Key Laboratory of Energy Genetics, Qingdao Institute of Bioenergy and Bioprocess Technology, Chinese Academy of Sciences, Qingdao 266101, Shandong, China. ${ }^{2}$ Institute of Modern Physics, Chinese Academy of Sciences, Lanzhou 730000, Gansu, China. ${ }^{3}$ Key Laboratory of Biofuels, Qingdao Institute of Bioenergy and Bioprocess Technology, Chinese Academy of Sciences, Qingdao 266101, Shandong, China. ${ }^{4}$ Qingdao Engineering Laboratory of Single Cell Oil, Qingdao 266101, Shandong, China. ${ }^{5}$ University of Chinese Academy of Sciences, Beijing 100049, China.

Received: 5 October 2017 Accepted: 18 April 2018 Published online: 02 May 2018

\section{References}

1. Ji XJ, Ren LJ, Nie ZK, Huang H, Ouyang PK. Fungal arachidonic acid-rich oil: research, development and industrialization. Crit Rev Biotechnol. 2014;34(3): 197-214.

2. Zhang AH, Ji XJ, Wu WJ, Ren LJ, Yu YD, Huang H. Lipid fraction and intracellular metabolite analysis reveal the mechanism of arachidonic acidrich oil accumulation in the aging process of Mortierella alpina. J Agric Food Chem. 2015;63(44):9812-9.

3. Kenichi Higashiyama SF, Enoch Y. Park, Sakayu Shimizu. Production of arachidonic acid by Mortierella Fungi. Biotechnol Bioprocess Eng. 2002;7: 252-62.

4. Nie ZK, Deng ZT, Zhang AH, Ji XJ, Huang H. Efficient arachidonic acid-rich oil production by Mortierella alpina through a three-stage fermentation strategy. Bioprocess Biosyst Eng. 2014;37(3):505-11.

5. Li X, Lin Y, Chang M, Jin Q, Wang X. Efficient production of arachidonic acid by Mortierella alpina through integrating fed-batch culture with a two-stage pH control strategy. BioresourTechnol. 2015;181:275-82.

6. Kikukawa H, Sakuradani E, Ando A, Okuda T, Ochiai M, Shimizu S, Ogawa J. Disruption of lig4 improves gene targeting efficiency in the oleaginous fungus Mortierella alpina 1S-4. J Biotechnol. 2015;208:63-9.

7. Ando A, Sakuradani E, Horinaka K, Ogawa J, Shimizu S. Transformation of an oleaginous zygomycete Mortierella alpina 1S-4 with the carboxin resistance gene conferred by mutation of the iron-sulfur subunit of succinate dehydrogenase. Curr Genet. 2009;55(3):349-56.

8. Takeno S, Sakuradani E, Tomi A, Inohara-Ochiai M, Kawashima H, Shimizu S. Transformation of oil-producing fungus, Mortierella alpina 1S-4, using Zeocin, and application to arachidonic acid production. J Biosci Bioeng. 2005;100(6):617-22.

9. Sakuradani E. Advances in the production of various polyunsaturated fatty acids through oleaginous fungus Mortierella alpina breeding. Biosci Biotechnol Biochem. 2010;74(5):908-17.

10. Liang MH, Jiang JG. Advancing oleaginous microorganisms to produce lipid via metabolic engineering technology. Prog Lipid Res. 2013;52(4):395-408.

11. Li Z, Chen X, Li Z, Li D, Wang Y, Gao H, Cao L, Hou Y, Li S, Liang J. Strain improvement of Trichoderma viride for increased cellulase production by irradiation of electron and ${ }^{12} \mathrm{C}^{6+}$-ion beams. Biotechnol Lett. 2016;38(6):983-9.

12. Cheng YR, Sun ZJ, Cui GZ, Song XJ, Cui Q. A new strategy for strain improvement of Aurantiochytrium sp. based on heavy-ions mutagenesis and synergistic effects of cold stress and inhibitors of enoyl-ACP reductase. Enzyme Microb Tech. 2016;93-94:182-90.

13. Yang S, Wang SY, Jiang BL, Zhou X, Chen JH, Li WJ, Liu J, Hu W, Xiao GQ, Dong MY, et al. Study of a high-yield Cellulase system created by heavy-ion irradiation-induced mutagenesis of Aspergillus niger and mixed fermentation with Trichoderma reesei. PLoS One. 2015;10(12):e0144233.

14. Williams K, Sobol RW. Mutation research/fundamental and molecular mechanisms of mutagenesis special issue: DNA repair and genetic instability. Mutat Res-Fund Mol M. 2013;743:1-3.

15. Baba M, Kikuta F, Suzuki I, Watanabe MM, Shiraiwa Y. Wavelength specificity ofgrowth, photosynthesis, and hydrocarbon production in the oil-producing green alga Botryococcusbraunii. BioresourTechnol. 2012;109:266-70.

16. Przybyła T, Sakowicz-Burkiewicz M, Maciejewska I, Bielarczyk H, Pawełczyk T. Suppression of ID1 expression in colon cancer cells increases sensitivity to 5-fluorouracil. Acta Biochim Pol. 2017;64(2):315-22.

17. Anderson NM, Berberovic Z, Berndl E, Bailey ML, Flenniken AM, Osborne LR, Adamson SL, Rossant J, Wang C, Minden MD, et al. Cytopenia induction by 5-fluorouracil identifies thrombopoietic mutants in sensitized ENU mutagenesis screens. Exp Hematol. 2012;40(1):48-60.
18. Wang L, Chen W, Feng Y, Ren Y, Gu ZN, Chen HQ, Wang HC, Thomas MJ, Zhang BX, Berquin IM, et al. Genome characterization of the oleaginous fungus Mortierella alpina. PLoS One. 2011;6(12):e28319. https://doi.org/10. 1371/journal.pone.0028319.

19. Vongsangnak W, Ruenwai R, Tang X, Hu X, Zhang H, Shen B, Song Y, Laoteng K. Genome-scale analysis of the metabolic networks of oleaginous Zygomycete fungi. Gene. 2013;521(1):180-90.

20. Twanabasu BR, Stevens KJ, Venables BJ. The effects of triclosan on spore germination and hyphal growth of the arbuscular mycorrhizal fungus Glomus intraradices. Sci Total Environ. 2013;454:51-60.

21. Wang Q, Zhang Y, Li H. Octyl gallate: an antioxidant demonstrating selective and sensitive fluorescent property. Food Chem. 2017;219:268-73.

22. Lakshmana MK, Zhang S-Q, Sawmiller D, Li S, Rezai-Zadeh K, Hou H, Zhou S, Shytle D, Giunta B, Fernandez F, et al. Octyl Gallate markedly promotes antiAmyloidogenic processing of APP through estrogen receptor-mediated ADAM10 activation. PLoS One. 2013;8(8):e71913.

23. Törmäkangas $L$, Vuorela $P$, Saario $E$, Leinonen $M$, Saikku $P$, Vuorela $H$. In vivo treatment of acute chlamydia pneumoniae infection with the flavonoids quercetin and luteolin and an alkyl gallate, octyl gallate, in a mouse model. Biochem Pharmacol. 2005:70(8):1222-30.

24. Hsu F, Chang H, Chang S. Evaluation of antifungal properties of octyl gallate and its synergy with cinnamaldehyde. Bioresour Technol. 2007:98(4):734-8.

25. Fujita K, Kubo I. Antifungal activity of octylgallate. Int J Food Microbiol. 2002; 79:193-201

26. Gao M, Song X, Feng Y, Li W, Cui Q. Isolation and characterization of Aurantiochytrium species: high docosahexaenoic acid (DHA) production by the newly isolated microalga, Aurantiochytrium sp. SD116. J Oleo Sci. 2013; 62(3):143-51.

27. Lukens L, Fitzgerald TL, Powell JJ, Stiller J, Weese TL, Abe T, Zhao G, Jia J, CL MI, $\mathrm{Li} Z$, et al. An assessment of heavy ion irradiation mutagenesis for reverse genetics in wheat (Triticum aestivum L.). PLoS One. 2015;10(2):e0117369.

28. Shi J, Lu W, Sun Y. Comparison of space flight and heavy ion radiation induced genomic/epigenomic mutations in rice (Oryza sativa). Life Sci Space Res. 2014;1:74-9.

29. Wang SY, Bo YH, Zhou X, Chen JH, Li WJ, Liang JP, Xiao GQ, Wang YC, Liu J, $\mathrm{Hu}$ W, et al. Significance of heavy-ion beam irradiation-induced Avermectin B1a production by engineered Streptomyces avermitilis. Biomed Res Int 2017;2017:1-13.

30. Li X, Liu R, Li J, Chang M, Liu Y, Jin Q, Wang X. Enhanced arachidonic acid production from Mortierella alpina combining atmospheric and room temperature plasma (ARTP) and diethyl sulfate treatments. BioresourTechnol. 2015:177:134-40.

31. Butler E, Whelan MJ, Ritz K, Sakrabani R, van Egmond R. The effect of triclosan on microbial community structure in three soils. Chemosphere. 2012:89(1):1-9.

32. Heath RJ, White SW, Rock CO. Inhibitors of fatty acid synthesis as antimicrobial chemotherapeutics. Appl Microbiol Biotechnol. 2002;58(6):695-703.

33. Cong LL, Ji XJ, Nie ZK, Peng C, Li ZY, Deng ZT, Huang H. Breeding of Mortierella alpina for high-yield arachidonic acid-rich oil production. Chinese Journal of Bioprocess Engineering. 2012;10(5):34-8.

34. Sage E, Shikazono N. Radiation-induced clustered DNA lesions: repair and mutagenesis. Free Radical Bio Med. 2017;107:125-35.

35. Ma Y, Wang Z, Zhu M, Yu C, Cao Y, Zhang D, Zhou G. Increased lipid productivity and TAG content in Nannochloropsis by heavy-ion irradiation mutagenesis. Bioresour Technol. 2013;136:360-7.

36. Boreyko AV, Krasavin EA. Mutagenic effect of accelerated heavy ions on bacterial cells. PhysPartNuclei. 2011;42(6):998-1024

37. Kawashima H, Akimoto K, Shirasaka N. Inhibitory effects of alkyl gallate and its derivatives on fatty acid desaturation. Biochim Biophy Acta. 1996;1299(1):34-8.

38. Zhang $H$, Feng $Y$, Cui Q, Song X. Expression of Vitreoscilla hemoglobin enhances production of arachidonic acid and lipids in Mortierella alpina. BMC Biotechnol. 2017:17(1):68.

39. Hao G, Chen H, Du K, Huang X, Song Y, Gu Z, Wang L, Zhang H, Chen W, Chen YQ. Increased fatty acid unsaturation and production of arachidonic acid by homologous over-expression of the mitochondrial malic enzyme in Mortierella alpina. Biotechnol Lett. 2014;36(9):1827-34.

40. Ji XJ, Zhang AH, Nie ZK, Wu WJ, Ren LJ, Huang H. Efficient arachidonic acidrich oil production by Mortierella alpina through a repeated fed-batch fermentation strategy. Bioresour Technol. 2014;170:356-60.

41. Nie ZK, Ji XJ, Shang JS, Zhang AH, Ren LJ, Huang H. Arachidonic acid-rich oil production by Mortierella alpina with different gas distributors. Bioprocess biosysteng. 2014;37(6):1127-32. 